\title{
APPLICATION OF MICROTEXTURE MEASUREMENTS IN THE SEM TO GRAIN BOUNDARY PARAMETERS
}

\author{
VALERIE RANDLE \\ Dept. of Materials Engineering, University College of Swansea, Swansea \\ SA2 8PP, U.K.
}

\begin{abstract}
This paper discusses how microtexture data, i.e. individual orientations which are measured on a grain and environmentally specific basis, are applied to grain boundary geometrical parameters. Three main areas are addressed: the "interface-plane" scheme for specifying the five degress of freedom of a boundary, comparisons of experimental techniques for data collection, and representation of grain boundary misorientations in Rodrigues-Frank space. Particular attention is paid to electron back-scatter diffraction as a method of probing grain boundary misorientation and the crystallographic orientation of the grain boundary plane.
\end{abstract}

KEY WORDS Grain boundary plane geometry, electron back-scatter diffraction, Rodrigues-Frank space, interface-plane scheme, coincidence site lattice.

\section{INTRODUCTION}

"Microtexture" is a word which has come to mean texture measured on the scale of the microstructure (Randle, 1992; Dingley and Randle, 1992). The essential feature of a microtexture is that it is composed of individual orientations which are usually spatially specific. These data unlock a wealth of information which is inaccessible by a conventional macrotexture approach. One example is that the grain boundary (GB) geometrical (i.e. crystallographic) parameters can be accessed (e.g. Furley and Randle, 1991). Individual orientations from contiguous grains can be re-expressed as misorientations, and, if additional spatial parameters are measured, the orientation of the GB plane can also be obtained (e.g. Randle, 1989; Randle and Dingley, 1989; Laws and Goodhew, 1991). Figure 1 shows schematically the relationship between macrotexture, microtexture and "mesotexture" or GB crystallography.

The reason for focussing attention on GB geometry is that GBs are active elements of the microstructure which influence its overall properties. Moreover, certain GBs are referred to as "special" because they are associated with properties or behaviour which is markedly different from average (e.g. Priester, 1989). Identification and study of such GBs is at the centre of much research activity, because their properties tend to be beneficial to the overall properties of the polycrystal.

The precise nature of "special" GBs is still unclear; the general picture is that special properties are governed by the crystallography, atomic-level structure, chemistry and electronic state of the GB (e.g. Priester, 1989; Sutton and Balluffi, 


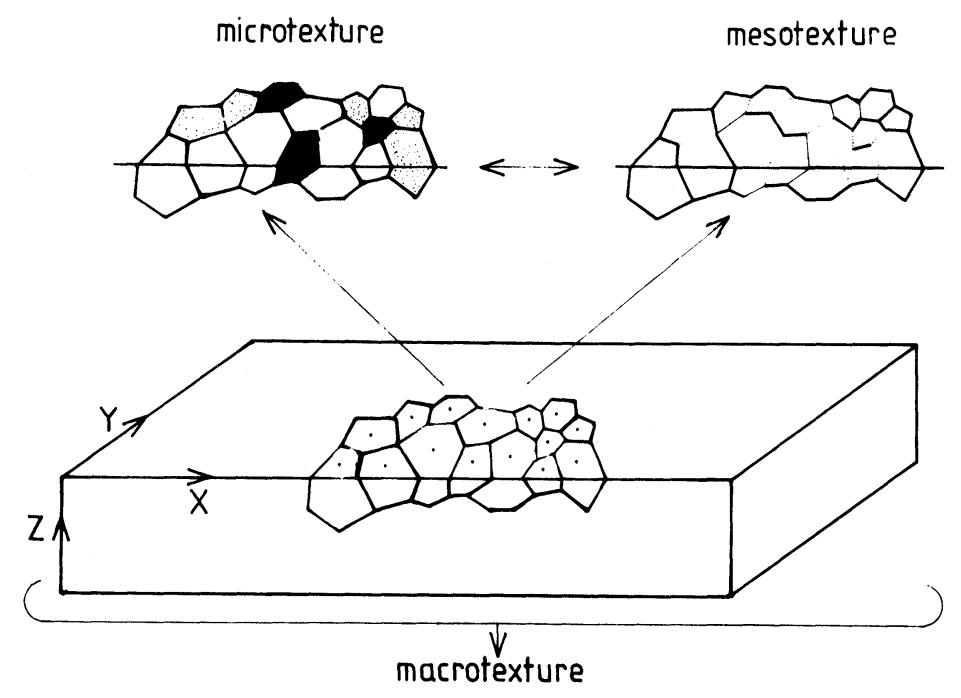

Figure 1 Schematic diagram illustrating the relationship between macrotexture (i.e. averaged over many grains), microtexture (i.e. measured on an individual grain basis) and mesotexture (i.e. the misorientation and grain boundary plane distribution).

1987). Of this list only GB crystallography (geometry) is easily accessible experimentally. However, it is the major component of GB structure and so is a valid guide to specialness. The coincidence site lattice (CSL) model is a convenient framework for the categorisation of GB geometry, and so has tended to persist as a tool for analysis in this field, despite the fact that its predictive power is not completely general, i.e. not all CSL GBs are special and vice versa. There are many examples in the literature of studies of GB geometry and its relationship to properties (e.g. Palumbo and Aust, 1989; Field and Adams, 1992).

For many years transmission electron microscopy (TEM) and selected area channelling (SAC) in the scanning electron microscope (SEM) were the main techniques used to obtain the GB parameters. Recently, electron back-scatter diffraction (EBSD) has superseded SAC as a more convenient SEM-based technique for microtexture measurements (Juul Jensen and Randle, 1989; Dingley and Randle 1992; Randle, 1992). The purpose of this report is to address three aspects of GB geometry: how to express the GB geometrical parameters, how to measure them and how to represent them (Neumann, 1991; Heinz and Neumann, 1991; Randle, 1992).

\section{DEGREES OF FREEDOM}

A fundamental description of the geometry of a GB is its degrees of freedom. A GB in a bicrystal has five macroscopic degrees of freedom, which can be defined collectively as the geometry which relates the overall orientation change across the GB plane. This definition emphasises the crystallographic nature of GB 

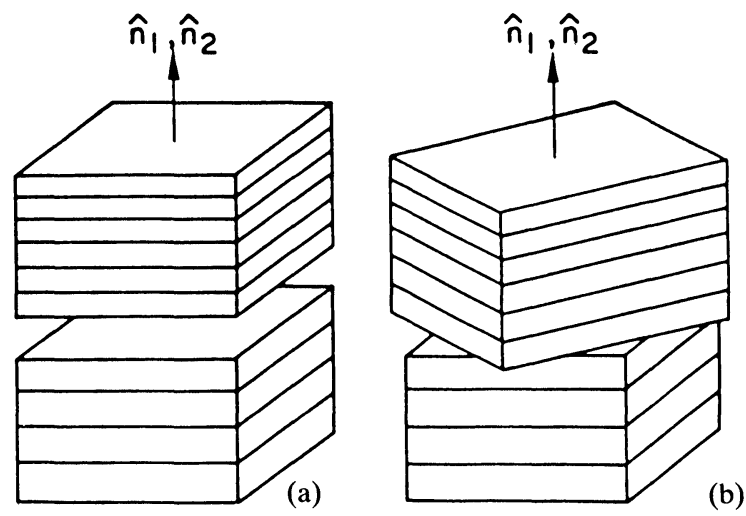

Figure 2 Illustration of the "interface-plane" scheme. (a) "Special" case where the GB normals from each grain are parallel and the twist angle between them is zero. (b) General case where the plane stacks from each grain are related by a twist rotation about the normal. (Courtesy of D. Wolf).

geometry. In addition, three further "extrinsic" degrees of freedom can be introduced which refer to a set of external axes, usually the specimen axes.

The "intrinsic" degrees of freedom are most commonly expressed as a misorientation between the two neighbouring lattices plus the orientation of the GB plane referred to the crystal axes of one grain. The misorientation is composed of the misorientation axis, $u v w$, and the misorientation angle, $\theta$. This formulation of the misorientation allows low angle GBs to be recognised directly, and also CSL classifications to be made. Frequently in an experiment the GB plane orientation is not measured, in which case the $\theta / u v w$ representation of the misorientation matrix is the most physically meaningful. It also allows the data to be plotted in Rodrigues-Frank space (see Section 4).

Where the GB plane orientation has been obtained the data become more powerful because the actual crystallography of the GB itself, rather than the neighbouring lattices, is known. For these cases it may be more relevant to express the five degrees of freedom using the interface-plane scheme (Wolf and Lutsko, 1989). The essential point about the interface plane scheme is that the emphasis is on formation of the GB by the joining of two lattice planes, plus a twist about their common normal, as shown in Figure 2. In this scheme a GB is denoted by the indices of the two adjoining surfaces of the GB, $n_{1}$ and $n_{2}$, plus the twist angle, $\phi$, which relates them.

A particular advantage of the interface plane scheme is that symmetrical tilt, asymmetrical tilt and twist GBs can be recognised readily. The conditions for each are:

1) Symmetrical tilt (STGB): $n_{1}=n_{2} ; \phi=0$. For example $[111][111] 0^{\circ}$ is a STGB in the $\Sigma=3$ CSL system.

2) Asymmetrical tilt (ATGB): $n_{1} \neq n_{2} ; \phi=0$. For example [110][114]0 is an ATGB in the $\Sigma=3$ system.

3) Twist (TWGB): $n_{1}=n_{2} ; \phi \neq 0$. For example [311][311]95.74 is a TWGB in the $\Sigma=5$ system. 
Table 1 Details of grain boundary geometries for CSL boundaries

\begin{tabular}{|c|c|c|c|c|c|c|c|}
\hline \multirow[b]{2}{*}{$\Sigma$} & \multicolumn{7}{|c|}{ "Slow" specimen } \\
\hline & $\begin{array}{l}\text { Relative } \\
\text { deviation }\end{array}$ & Planes & $w^{\circ}$ & $\Sigma$ & $\begin{array}{l}\text { Relative } \\
\text { deviation }\end{array}$ & Planes & $w^{\circ}$ \\
\hline & \multicolumn{4}{|c|}{ Asymmetrical tilts } & \multicolumn{3}{|c|}{ Symmetrical tilts } \\
\hline 3 & 0.17 & $111 / 511$ & 3.0 & 3 & 0.17 & $111 / 111$ & 5.6 \\
\hline 3 & 0.13 & $100 / 221$ & 4.1 & 3 & 0.10 & $111 / 111$ & 7.5 \\
\hline 3 & 0.30 & $100 / 211$ & 5.0 & 3 & 0.27 & $111 / 111$ & 6.6 \\
\hline 3 & 0.62 & $100 / 221$ & 5.5 & 9 & 0.39 & $411 / 411$ & 4.1 \\
\hline 3 & 0.28 & $100 / 221$ & 6.2 & & & & \\
\hline 3 & 0.16 & $110 / 411$ & 4.1 & & twists & & 7.0 \\
\hline 3 & 0.32 & $110 / 411$ & 7.4 & 3 & 0.04 & $210 / 210$ & 6.0 \\
\hline 3 & 0.08 & $110 / 411$ & 1.3 & 3 & 0.09 & $311 / 311$ & 2.5 \\
\hline 3 & 0.52 & $110 / 411$ & 3.3 & 3 & 0.45 & $311 / 311$ & 2.5 \\
\hline 3 & 0.32 & $110 / 411$ & 3.2 & 9 & 0.67 & $322 / 322$ & 6.1 \\
\hline 3 & 0.16 & $110 / 411$ & 4.3 & $27 \mathrm{a}$ & 0.21 & $411 / 411$ & \\
\hline 3 & 0.05 & $100 / 744$ & 5.5 & & & & \\
\hline 3 & 0.62 & $100 / 221$ & 4.1 & & & & \\
\hline 3 & 0.72 & $210 / 542$ & 3.7 & & & & \\
\hline 3 & 0.18 & $210 / 542$ & 2.1 & & \multicolumn{3}{|c|}{ Asymmetrical tilts } \\
\hline 3 & 0.55 & $110 / 11,54$ & 5.5 & 9 & 0.74 & $110 / 11,54$ & 5.7 \\
\hline 3 & 0.22 & $211 / 552$ & 3.5 & 9 & 0.57 & $210 / 17,10,4$ & 2.2 \\
\hline 3 & 0.41 & $211 / 721$ & 3.9 & 9 & 0.58 & $111 / 13,7,5$ & 6.3 \\
\hline 3 & 0.22 & $211 / 721$ & 6.3 & 9 & 0.74 & $111 / 511$ & 2.8 \\
\hline 3 & 0.19 & $211 / 721$ & 1.4 & 9 & 0.93 & $111 / 511$ & 4.2 \\
\hline 3 & 0.16 & $211 / 552$ & 4.8 & 9 & 0.25 & $311 / 771$ & 7.0 \\
\hline 3 & 0.44 & $221 / 744$ & 5.7 & $17 \mathrm{a}$ & 0.77 & $210 / 30,17,16$ & 2.8 \\
\hline 3 & 0.30 & $221 / 841$ & 3.1 & $19 a$ & 0.51 & $100 / 18,6,1$ & 4.0 \\
\hline 3 & 0.66 & $221 / 841$ & 4.3 & $19 b$ & 0.78 & $110 / 25,9,4$ & 2.5 \\
\hline 3 & 0.12 & $221 / 744$ & 1.6 & 11 & 0.28 & $311 / 31,17,9$ & 4.4 \\
\hline 3 & 0.14 & $221 / 744$ & 3.1 & & & & \\
\hline 3 & 0.20 & $311 / 755$ & 1.2 & & & & \\
\hline \multicolumn{8}{|c|}{ "Fast" specimen } \\
\hline$\Sigma$ & \multicolumn{4}{|c|}{ Asymmetric tilts } & \multicolumn{3}{|c|}{ Symmetrical tilts } \\
\hline 3 & 0.15 & $221 / 744$ & 5.2 & 3 & 0.24 & $111 / 111$ & 3.5 \\
\hline 3 & 0.17 & $110 / 411$ & 4.5 & 3 & 0.49 & $111 / 111$ & 5.4 \\
\hline 3 & 0.37 & $511 / 13,75$ & 6.4 & 3 & 0.33 & $211 / 211$ & 6.5 \\
\hline 3 & 0.25 & $331 / 11,71$ & 4.9 & 3 & 0.96 & $211 / 211$ & 5.9 \\
\hline 3 & 0.58 & $100 / 744$ & 5.7 & & & & \\
\hline 3 & 0.69 & $221 / 744$ & 5.7 & & & & \\
\hline 3 & 0.92 & $111 / 511$ & 4.1 & & twists & & \\
\hline 3 & 0.48 & $331 / 11,71$ & 5.1 & 3 & 0.88 & $210 / 210$ & 4.1 \\
\hline 3 & 0.16 & $311 / 755$ & 5.6 & 7 & 0.45 & $320 / 320$ & 4.4 \\
\hline 3 & 0.55 & $310 / 754$ & 5.5 & & & & \\
\hline $27 b$ & 0.83 & $210 / 40,37,26$ & 1.4 & & & & \\
\hline 7 & 0.75 & $310 / 20,93$ & 2.9 & & & & \\
\hline 7 & 0.99 & $221 / 19,84$ & 6.6 & & & & \\
\hline $17 \mathrm{a}$ & 0.85 & $100 / 15,80$ & & & & & \\
\hline
\end{tabular}


4) GBs which are general with respect to GB planes: $n_{1} \neq n_{2} ; \phi \neq 0$. For example [110][543] $26.52^{\circ}$ is a general GB in the $\Sigma=5$ system.

The CSL system can be recognised by the ratio of the separation distance of the lattice planes, which is in turn proportional to the ratio of the planar GB unit cells, $A_{1} / A_{2}$. For example, the general case above (no. 4) yields $A_{1} / A_{2}=$ $(50 / 2)^{1 / 2}=5$; thus this $\mathrm{GB}$ is in the $\Sigma=5$ system. The various relationships between the tilt and twist parameters and the misorientation scheme can be found elsewhere (Wolf and Lutsko, 1989).

Table 1 shows an example of a set of experimental GB geometrical datamisorientation plus GB plane-obtained from annealed nickel (Randle, 1992). Only the CSL GBs are included, and the table includes the $\Sigma$-value (column 1), the relative deviation away from exact CSL matching (column 2), the nearest low-index GB planes (column 3) and the angular deviation of the exact plane orientation (averaged for the two grains) away from the nearest low-index plane (column 4). These data were measured using the EBSD "corner GB" method (see Section 3).

\section{EXPERIMENTAL METHODS FOR MEASURING GRAIN BOUNDARY PARAMETERS}

Although some X-ray based techniques can be used to measure the GB geometrical parameters (e.g. synchrotron radiation) invariably an electron beam method is chosen because local diffraction and imaging of the associated region can be performed concurrently. The parameters can be obtained using either TEM or SEM (EBSD); the EBSD route is more efficient both in terms of specimen preparation and data collection. However, other factors have to be taken into account when choosing the most suitable route for a particular investigation. These are:

1) A diffraction capability is an inherent part of the TEM, because of the way in which the image is formed. Consequently GB crystallography can be measured with a standard TEM, preferably one which has a diffraction capability. On the other hand additional equipment has to be added to a standard SEM before diffraction can be performed. A typical EBSD system consists of a low-light TV camera interfaced to a phosphor screen, a camera control unit, monitor and computer plus dedicated software.

2) If both TEM and EBSD are available, often the factor which influences the choice is resolution (Schwarzer, 1990). The resolution for EBSD is approximately $500 \mathrm{~nm}$ diameter whereas for TEM it is an order of magnitude smaller. Hence very fine grain sized materials require TEM. A further consequence of the size of the sample volume relates to cold work. A certain amount of lattice strain can be tolerated in EBSD patterns, so that for instance the technique can be used for measuring the GB crystallography across a recrystallisation interface where either the level of cold work is fairly small, or the dislocation substructure has formed cells such that the probe can be sited between the cell walls. The much smaller probe in TEM enables diffraction patterns to be obtained from regions with much higher levels of cold work. 


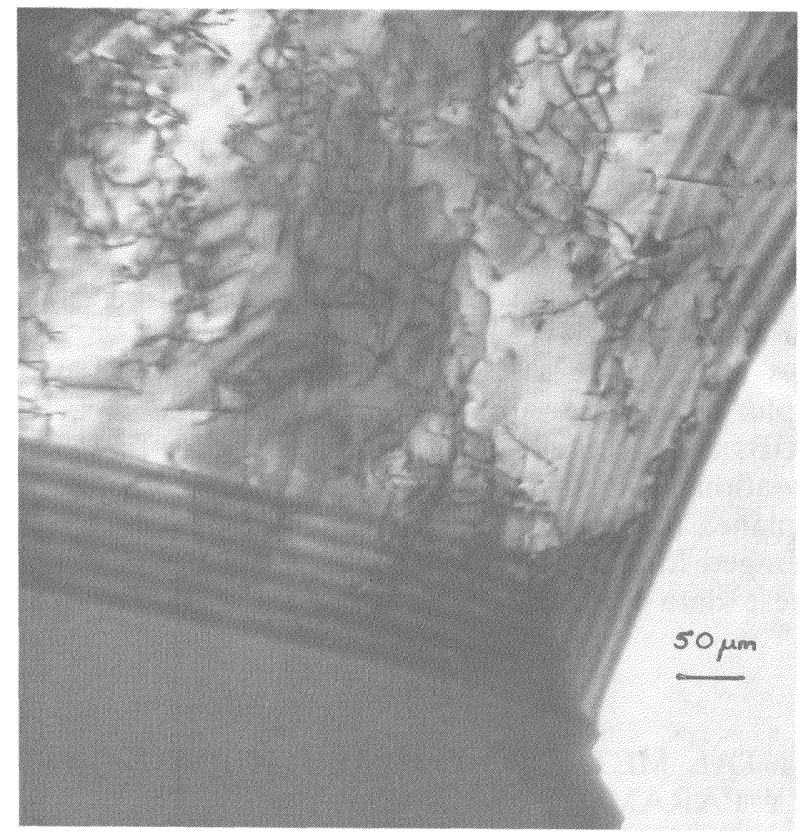

Figure 3 Three GBs meeting at a triple point in partially recrystallised steel. Two of the GBs are suitable for plane normal determination by the "foil thickness" method because their projected width is relatively large.

3) TEM provides a direct image of the fine detail in the microstructure, such as dislocations, which in the SEM can only be inferred. Where the GB parameters are to be related to external axes, however, SEM is the better choice because it is much easier to define axes on a bulk specimen than on a thin foil.

4) In order to calculate a misorientation, the orientation of two adjacent grain must be measured, relative to a common reference frame. This procedure is performed on-line for EBSD, with some operator input. For TEM the route is either to obtain photographs of the diffraction patterns and analyse them off-line, or alternatively the relevant input parameters can be extracted from the "live" diffraction patterns and the analysis performed on-line. Obtaining a GB plane orientation requires more input data in both cases; these are discussed in the next subsection.

5) The accuracy of the TEM and EBSD method for obtaining a misorientation is $0.2^{\circ}$ and $0.5^{\circ}$ respectively. The principal source of error in the EBSD case is specification of the pattern centre, PC. In a TEM diffraction pattern the PC is concentric with the beam and so coincides with the physical centre of the pattern. For EBSD the position of the PC is governed by the point where the electron beam impinges on the specimen, and is determined by a calibration routine. Recently there have been improvements in the EBSD calibration routine; these are documented elsewhere (Dingley and Randle, 1992; Randle, 1992). 


\subsection{Determination of Grain Boundary Plane Orientation}

The determination of the GB plane orientation, which is essential for a complete description of all five degrees of freedom, requires a knowledge of the inclination of the GB within the specimen. In the TEM this parameter, and hence the GB plane orientation, can be obtained by various tilting experiments (e.g. Laws and Goodhew, 1991) or by a method which incorporates measurements of the foil thickness (Randle, 1989). Foil thickness can be measured readily using a convergent beam electron diffraction (CBED) technique. For maximum accuracy a GB with a large projected width is required as in Figure 3.

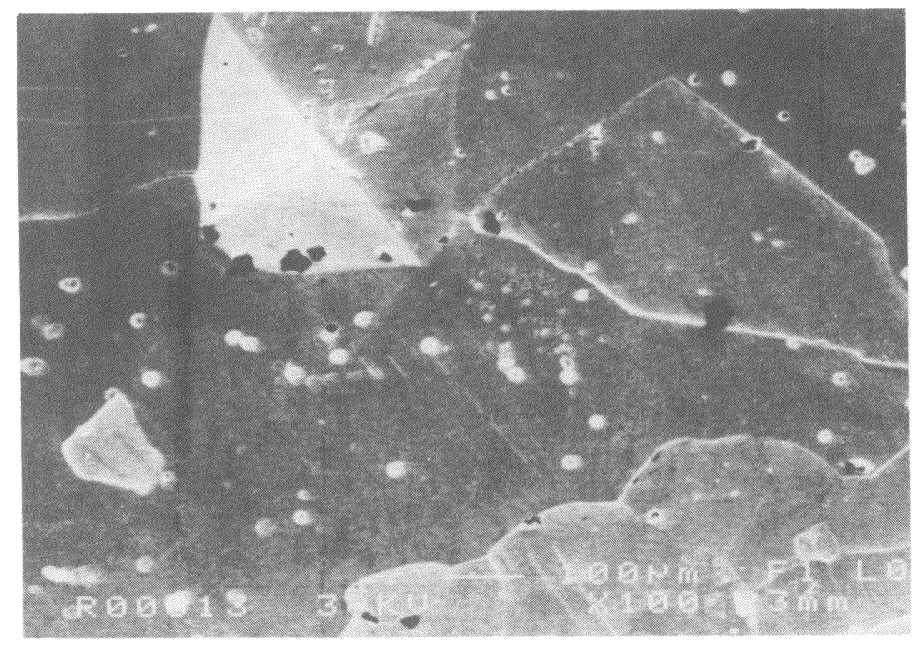

(a)

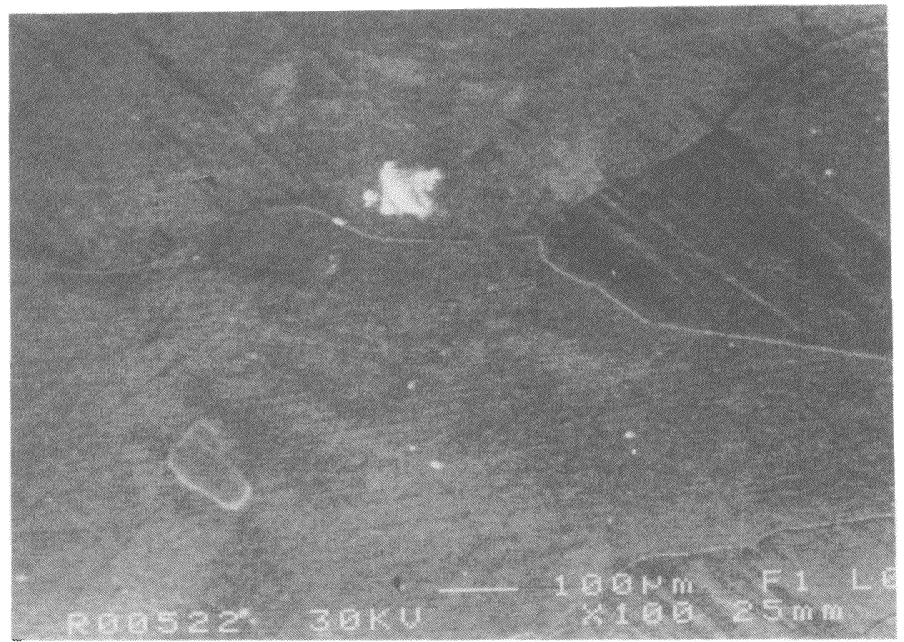

(b)

Figure 4 GB plane normal determination in the SEM by a serial sectioning method. (a) Position of GBs relative to a hardness indent. (b) Position of GBs and hardness indent after removing $50 \mu \mathrm{m}$ depth by grinding and polishing. 
There are at least two SEM-based methods for obtaining the GB orientation which can be used on a bulk specimen. One relies on a serial sectioning technique to obtain the GB inclination, using a hardness indent to measure the depth of surface removed. The inclination is calculated from the position of the GB trace before and after sectioning; Figure 4 shows an example. A second method uses a "two-surface trace analysis" approach (Randle and Dingley, 1989). Here the angle of the GB trace is measured on two mutually perpendicular specimen surfaces. The reference direction for the angular measurements is the specimen edge which is common to the two surfaces; this direction is made to lie accurately perpendicular to the horizontal axis in the microscope which in turn is parallel to the horizontal axis in the diffraction pattern. Figure 5a illustrates these experimental arrangements on a micrograph of a typical "two-surface" specimen, accompanied by a schematic drawing (Figure $5 b$ ).
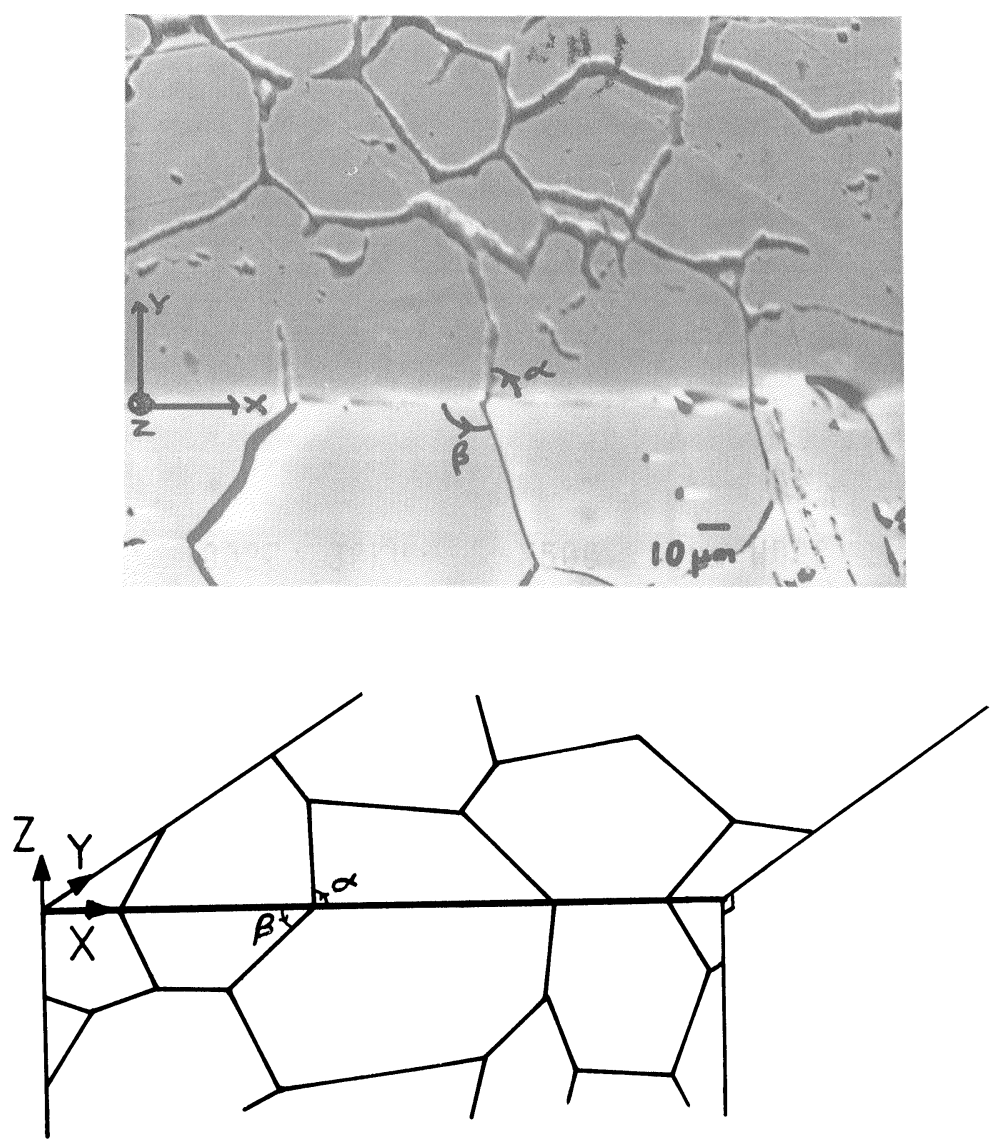

Figure 5 (a) GB plane normal determination in the SEM by a "two-surface" method. The micrograph shows two mutually perpendicular faces of a nickel specimen which has been tilted so as to photograph both faces together. The $X, Y$ and $Z$ direction in the specimen are marked. (b) Schematic illustration of the "two surface" method. 

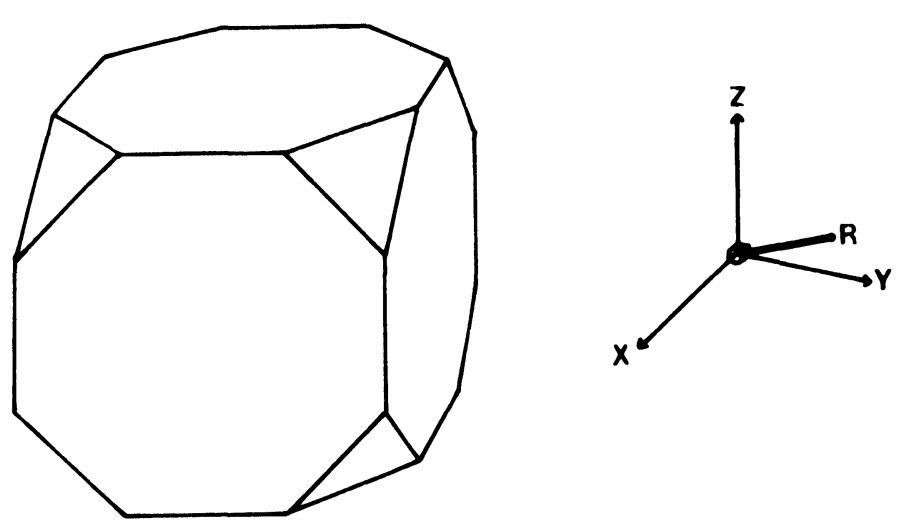

Figure 6 The fundamental zone of RF space. The origin of the $X Y Z$ axes and the Rodrigues vector $R$ are at the centre of the zone.

\section{REPRESENTATION OF GRAIN BOUNDARY PARAMETERS}

If specification of external axes is included, an eight parameter space is needed to represent the GB parameters. This requirement is met by using two-dimensional projections through the space (Field and Adams, 1992). However frequently not all of these parameters are measured; the misorientation is often identified as the principal parameter set and in these cases representation of the GB parameters reduces to representation of the misorientation. It is a requirement of any

Table 2 Components $R_{1} R_{2} R_{3}$ of the $R$ vector for CSLs having $\Sigma$-values up to 45 , grouped according to for the lowest angle solution

\begin{tabular}{llllllllllll}
\hline 100 & & & & 110 & & & & 111 & & & \\
5 & $1 / 3$ & 0 & 0 & 9 & $1 / 4$ & $1 / 4$ & 0 & 3 & $1 / 3$ & $1 / 3$ & $1 / 3$ \\
$13 \mathrm{a}$ & $1 / 5$ & 0 & 0 & 11 & $1 / 3$ & $1 / 3$ & 0 & 7 & $1 / 5$ & $1 / 5$ & $1 / 5$ \\
$17 \mathrm{a}$ & $1 / 4$ & 0 & 0 & $19 \mathrm{a}$ & $1 / 6$ & $1 / 6$ & 0 & $13 \mathrm{~b}$ & $1 / 7$ & $1 / 7$ & $1 / 7$ \\
$25 \mathrm{a}$ & $1 / 7$ & 0 & 0 & $27 \mathrm{a}$ & $1 / 5$ & $1 / 5$ & 0 & $19 \mathrm{~b}$ & $1 / 4$ & $1 / 4$ & $1 / 4$ \\
$29 \mathrm{a}$ & $2 / 5$ & 0 & 0 & $33 \mathrm{a}$ & $1 / 8$ & $1 / 8$ & 0 & $21 \mathrm{a}$ & $1 / 9$ & $1 / 9$ & $1 / 9$ \\
$37 \mathrm{a}$ & $1 / 6$ & 0 & 0 & $33 \mathrm{c}$ & $2 / 5$ & $2 / 5$ & 0 & $31 \mathrm{a}$ & $1 / 11$ & $1 / 11$ & $1 / 11$ \\
$41 \mathrm{a}$ & $1 / 9$ & 0 & 0 & $41 \mathrm{c}$ & $3 / 8$ & $3 / 8$ & 0 & $37 \mathrm{c}$ & $3 / 11$ & $3 / 11$ & $3 / 11$ \\
& & & & & & & & $39 \mathrm{a}$ & $1 / 6$ & $1 / 6$ & $1 / 6$ \\
& & & & & & & & $43 \mathrm{a}$ & $1 / 13$ & $1 / 13$ & $1 / 13$ \\
210 & & & & 211 & & & & 221 & & & \\
15 & $2 / 5$ & $1 / 5$ & 0 & $21 \mathrm{~b}$ & $2 / 6$ & $1 / 6$ & $1 / 6$ & $17 \mathrm{~b}$ & $2 / 5$ & $2 / 5$ & $1 / 5$ \\
$27 \mathrm{~b}$ & $2 / 7$ & $1 / 7$ & 0 & $31 \mathrm{~b}$ & $2 / 5$ & $1 / 5$ & $1 / 5$ & $29 \mathrm{~b}$ & $2 / 7$ & $2 / 7$ & $1 / 7$ \\
$41 \mathrm{~b}$ & $2 / 6$ & $1 / 6$ & 0 & $35 \mathrm{a}$ & $2 / 8$ & $1 / 8$ & $1 / 8$ & $45 \mathrm{~b}$ & $2 / 9$ & $2 / 9$ & $1 / 9$ \\
$43 \mathrm{~b}$ & $2 / 9$ & $1 / 9$ & 0 & & & & & $45 \mathrm{c}$ & $2 / 6$ & $2 / 6$ & $1 / 6$ \\
& & & & & & & & & & & \\
310 & & & & 311 & & & & 331 & & & \\
$37 \mathrm{~b}$ & $3 / 8$ & $1 / 8$ & 0 & 23 & $3 / 9$ & $1 / 9$ & $1 / 9$ & $25 \mathrm{~b}$ & $3 / 9$ & $3 / 9$ & $1 / 9$ \\
& & & & $33 \mathrm{~b}$ & $3 / 11$ & $1 / 11$ & $1 / 11$ & $35 \mathrm{~b}$ & $3 / 11$ & $3 / 11$ & $1 / 11$ \\
321 & & & & & & & & & & & \\
$39 \mathrm{~b}$ & $3 / 8$ & $2 / 8$ & $1 / 8$ & & & & & $43 \mathrm{c}$ & $3 / 8$ & $3 / 8$ & $2 / 8$ \\
\hline
\end{tabular}


representation that departures from randomness should be clearly recognisable. In the context of misorientations, these include low angle GBs and low- $\Sigma$ CSLs.

Misorientations displayed in Rodrigues-Frank (RF) space show clearly low angle (LA), CSL and coincidence axial direction (CAD) GBs, and the locations of these GBs in RF space will now be demonstrated. The Rodrigues vector is defined as (Frank, 1988).

$$
R=u v w \tan (\theta / 2)
$$

If the form of $\theta / u v w$ which contains the lowest $\theta$ is used, all the $R$-vectors lie in a space known as the fundamental zone (Figure 6). In order to represent

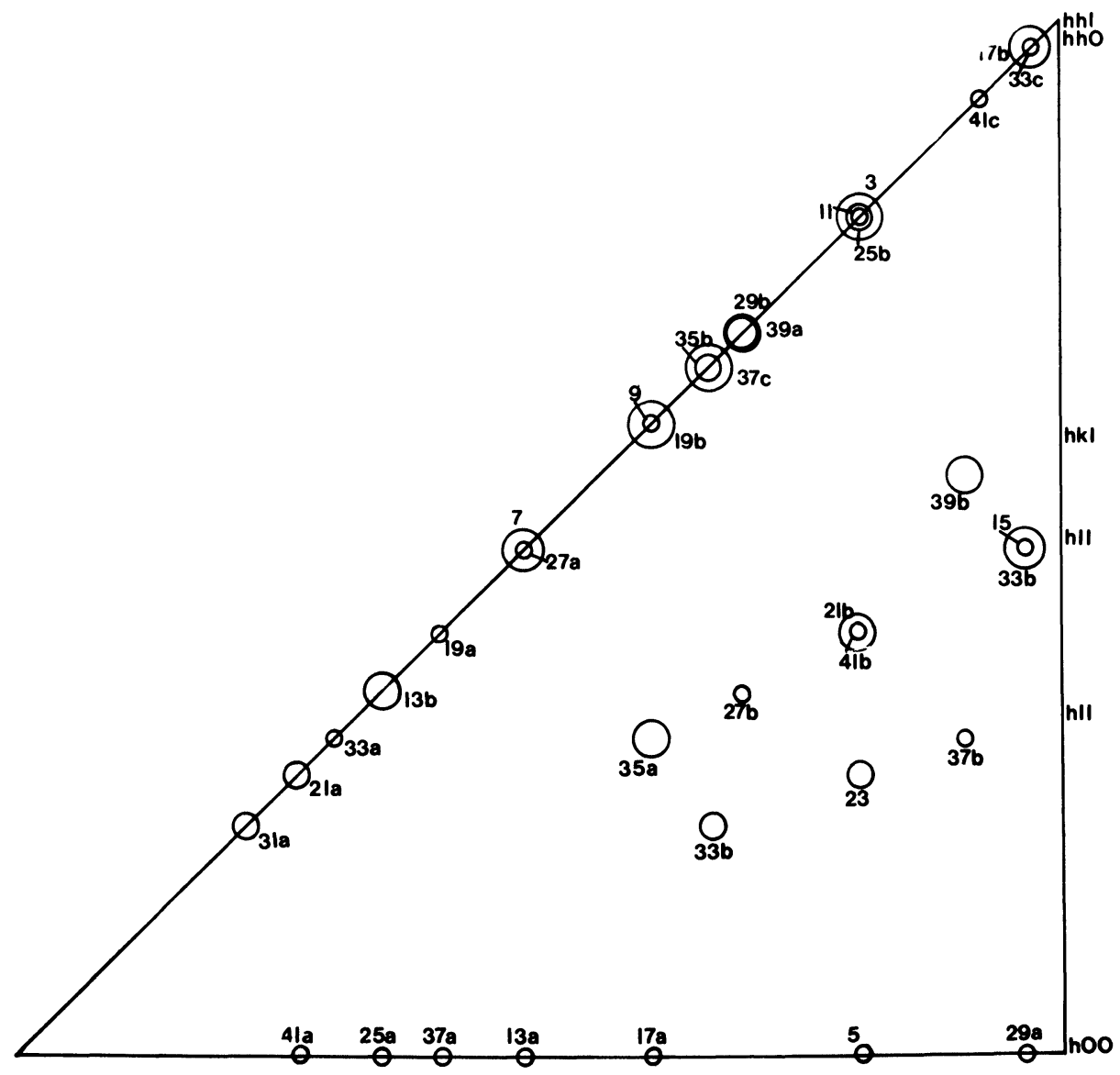

Figure 7 Location of CSLs with $\Sigma$ up to 41 in a projection of $1 / 48$ of the fundamental zone. The projection plane is part of the $X Y$ plane. The size of the symbols used to indicate $R$-vectors of CSLs is proportional to their $Z$-component (see Table 2).*

* The smallest symbols denote CSLs which are actually in the $X Y$ plane $(Z=0)$, and the largest symbols denote the largest $Z$-component. 
misorientations, only $1 / 48$ th of the fundamental zone, the subvolume, is required. This is analogous to a single unit triangle of the stereogram. It is striking that the components of the $R$-vectors for low- $\Sigma$ CSLs are rational fractions, with the misorientation axis given by the numerators (Randle, 1990). The $R$-vectors for CSLs having $\Sigma$-values up to 45 are listed in Table 2 according to misorientation axis. The actual location of these CSLs in a projection of 1/48th of the fundamental zone is shown in Figure 7. Conveniently, the subvolume can be sectioned parallel to its triangular "base" for display in two-dimensional sections. Figure 8 shows an experimental example from an annealed austenitic steel where clusters corresponding to several CSLs are apparent, and also LA GBs and some clustering around the 210-310 CAD axes which are marked on Figure 8.
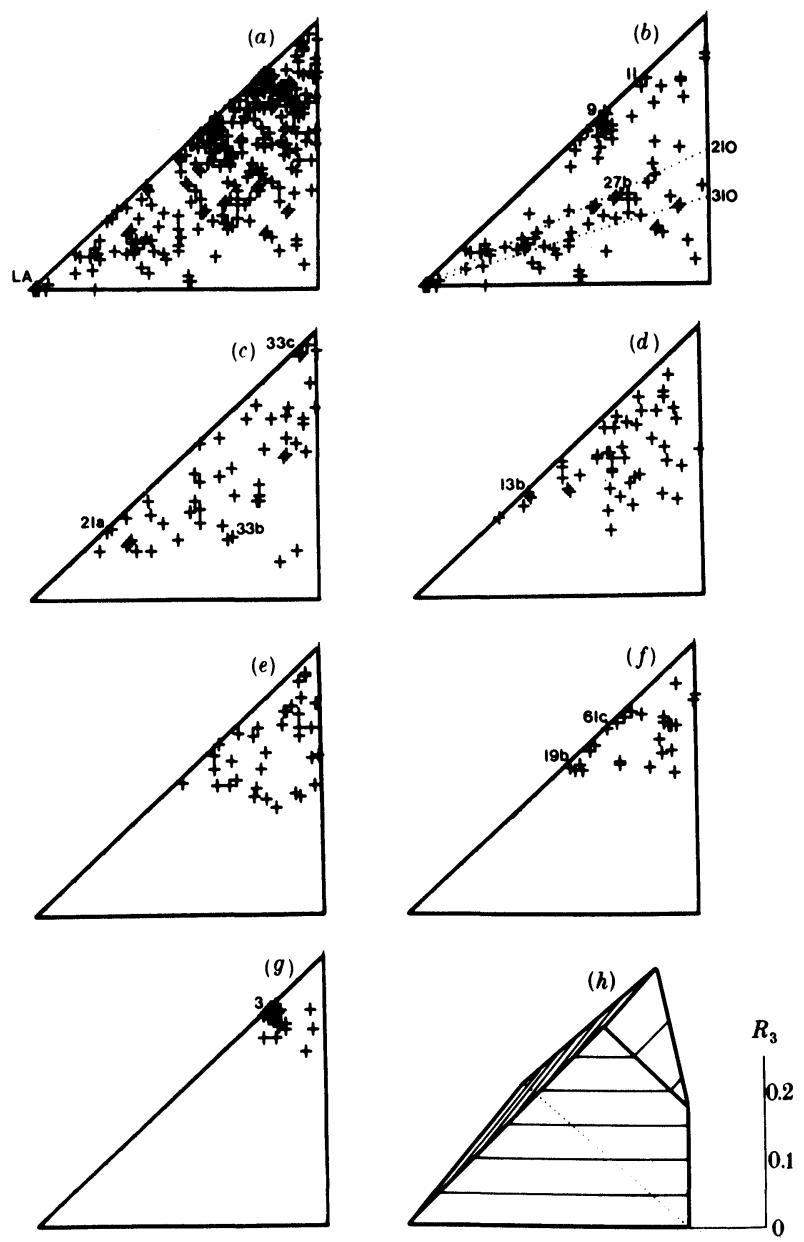

Figure 8 An experimental set of misorientations from an annealed steel displayed in 1/48 RF space. (a) is a projection of all the data points, (b)-(f) are sections through the subvolume as indicated in (h). Some CSLs are marked and also two CAD axes and LA GBs. 


\section{Acknowledgements}

The author is grateful to the Royal Society for financial support via a University Research Fellowship.

\section{References}

Dingley, D. J. and Randle, V. (1992). Microtexture determination by electron back-scatter diffraction-a review. J. Mat. Sci., in press.

Field, D. P. and Adams, B. L. (1992). Interface cavitation damage in polycrystalline copper. Acta Met. Mat., 40, 1145-1154.

Frank, F. C. (1988). Orientation mapping. Materials Research Society Bulletin, March 1988, 24-30.

Furley, J. and Randle, V. (1991). Mesotexture in annealed nickel. Mat. Sci. Tech., 7, 12-19.

Heinz, A. and Neumann, P. (1991). Representation of orientation and disorientation data of cubic, hexagonal, tetragonal and orthorhomic crystals. Acta Cryst., A47, 780-789.

Juul Jensen, D. and Randle, V. (1989). Combined advanced techniques in the study of annealing processes. In Proc. 10th Risø Int. Symp., Materials Architecture, edited by J. B. Bilde-Sorensen et al., Ris $\emptyset$ press, Denmark, 103-126.

Laws, M. S. and Goodhew, P. J. (1991). Grain boundary structure and chromium segregation in a 316 stainless steel. Acta Met. Mat., 39, 1525-1533.

Neumann, P. (1991). The representation of symmetrical objects by Rodrigues Vectors. Tex. and Micros., 14-18, 53-58.

Palumbo, G. and Aust, K. T. (1990). Structure-dependence of intergranular corrosion in high purity nickel. Acta Met. Mat., 11, 2343-2352.

Priester, L. (1989). Geometrical speciality and special properties of grain boundaries. Revue. Phys. Appl., 24, 419-438.

Randle, V. (1989). The distribution of grain boundary plane orientations in a metastable grain aggregate. Scripta Met., 23, 773-778.

Randle, V. (1990). The representation of grain misorientations (mesotexture) in Rodrigues-Frank space. Proc. Roy. Soc., 413A, 61-69.

Randle, V. (1992). Influence of kinetic factors on the distribution of grain boundary planes in nickel. Mat. Sci. Tech., 7, 985-990.

Randle, V. (1992). Microtexture determination and its applications, Institute of Materials, London.

Randle, V. and Dingley, D. J. (1989). Measurement of boundary plane inclination in a scanning electron microscope. Scripta Met., 23, 1565-1570.

Randle, V. (1992). Grain boundary geometry in cubic polycrystals, Institute of Physics Publishing, Bristol, in press.

Schwarzer, R. A. (1990). Measurement of local textures with transmission and scanning electron microscopes. Tex. Micros., 13, 15-30.

Sutton, A. P. and Balluffi, R. W. (1987). On geometric criteria for low interfacial energy. Acta Met., 35, 2177-2201.

Wolf, D. and Lutsko, J. F. (1989). On the geometrical relationship between tilt and twist grain boundaries. Zeit. f. Krist., 189, 239-262. 